\title{
Spectrum Reuse Schemes with Power Control for Device-to-Device Communication in LTE-Advanced Cellular Network
}

\author{
Sok Chhorn, Seok-Ho Yoon, Si-O Seo, Seung-Yeon Kim, and *Choong-Ho Cho \\ Department of Computer and Information Science, Korea University, Sejong, Korea \\ [e-mail: \{chhorn168, bluepig5, sioseo, kimsy8011, chcho\}@korea.ac.kr] \\ *Corresponding author: Choong-Ho Cho
}

Received August 11, 2015; revised September 17, 2015; accepted October 18, 2015; published December 31, 2015

\begin{abstract}
The spectral efficiency of cellular networks can be improved when proximate users engage in device-to-device (D2D) communications to communicate directly without going through a base station. However, D2D communications that are not properly designed may generate interference with existing cellular networks. In this paper, we study resource allocation and power control to minimize the probability of an outage and maximize the overall network throughput. We investigate three power control-based schemes: the Partial Co-channel based Overlap Resource Power Control (PC.OVER), Fractional Frequency Reuse based Overlap Resource Power Control (FFR.OVER) and Fractional Frequency Reuse based Adaptive Power Control (FFR.APC) and also compare their performance. In PC.OVER, a certain portion of the total bandwidth is dedicated to the D2D. The FFR.OVER and FFR.APC schemes combine the FFR techniques and the power control mechanism. In FFR, the entire frequency band is partitioned into two parts, including a central and edge sub-bands. Macrocell users (mUEs) transmit using uniform power in the inner and outer regions of the cell, and in all three schemes, the D2D receivers (D2DRs) transmit with low power when more than one D2DRs share a resource block (RB) with the macrocells. For PC.OVER and FFR.OVER, the power of the D2DRs is reduced to its minimum, and for the FFR.APC scheme, the transmission power of the D2DRs is iteratively adjusted to satisfy the signal to interference ratio (SIR) threshold. The three schemes exhibit a significant improvement in the overall system capacity as well as in the probability of a user outage when compared to a conventional scheme.
\end{abstract}

Keywords: LTE-Advanced, interference avoidance, Device-to-Device communications, Fractional Frequency Reuse, resource allocation, power control 


\section{Introduction}

Recently, the amount of traffic that flow through cellular networks has increased as a result of the growing popularity of mobile multimedia services. To meet the future requirements of the market, including local area optimization, new technologies have been investigated beyond Long Term Evolution-Advanced (LTE-A) [1]. Device-to-device (D2D) communication has been determined as a candidate technology for further study in Third Generation Partnership Project (3GPP) Release 12 [2]. Furthermore, for 3GPP LTE-A and future cellular networks, D2D communications have been proposed to facilitate direct communications among devices without the involvement of a base stations or the intervention of wireless operators [3]. Exploiting direct communication between nearby mobile devices will improve spectrum utilization, overall throughput, and energy consumption, while enabling new Peer-to-Peer (P2P) and location-based applications and services. In terms of conceptual and theoretical model, D2D communication can be seen as an extension of the concept of Cognitive Radio and dynamic spectrum access, where the ordinary cellular links can be seen as primary links and D2D as secondary links. The difference with the cognitive radio model is that the primary system is aware and supportive about the secondary links [4].

Meanwhile, due to the rapid development of mobile communication technologies, more and more users tend to download content to their mobile devices. This results in macro base stations (mBS) handling more traffic than in the past due to the fast-growing requirements for high data rate services. In Ref. [5], the authors proposed handling local P2P traffic in a reliable, scalable, and cost-effective manner by enabling direct D2D communication as an underlay to an IMT Advanced cellular network.

Refs. [6] and Ref. [7] studied the mode selection for spectrum sharing between D2D links and cellular UEs in wireless networks. To satisfy the interference constrain from the enable D2D communication in TV white space and the licensed digital TV services, the spectrum sharing between D2D links are considered under the cellular network [8]. However, in this paper, we do not consider D2D communication in TV white space. Ref. [9] considered the power control in D2D communications in order to minimize the total transmission power in the downlink (DL) subject to UE quality of service (QoS) demands. D2D communication can offload the traffic handled by the mBS and can reduce the end-to-end transmission delay since end users are able to exchange data directly without the intervention of mBS. However, D2D links may generate a high level of interference for macrocell users (mUEs) who are located within range of their communication if the D2D links transmit data using the same spectrum as the mUEs [10]. Thus, Ref. [11] presents a study on interference management in D2D to improve reliability. In addition, some state-of-art interference management mechanisms for D2D networks have been discussed in Refs. [12]-[13].

\section{A. Related Work and Contribution}

We initially conducted an experiment to examine the interference between D2D communications and cellular systems. The experimental environment consisted of a single-cell environment with 200 D2D links, with 20W of power for the mBS' transmission (Tx) and $6.3 \mathrm{~mW}$ and $251 \mathrm{~mW}$ of Tx power for the D2D server (D2DS). The results of the experiment indicate that the signal to interference and noise ratio (SINR) values that were received by the mUE decreased and that the mUE of the cell edge region receives almost no service (i.e. an outage ratio below $-6 \mathrm{~dB},[14]$ ). To address this problem, researchers have 
investigated methods to reduce the interference between D2D receivers (D2DRs) and mUEs in cellular networks supporting D2D communication. With respect to earlier studies, the authors of Ref. [10] suggested using the channels of the D2D links first if the frequency bands were not already being utilized in the mBS, and the channel status of the D2D links is observed to determine whether all frequency bands are being used and assigned to the best channel from the mBS. Furthermore, Ref. [7] introduced a technique to measure one's own channel gain and the channel gain till the mUE uses an applicable channel, and Ref. [15] suggested assigning the channel with the lowest gain to the D2D terminal. However, such studies have focused on the interference control at the cell center and do not improve the performance of the mUEs at the edge of the cell. In addition, these are not designed to respond to strong signals that have been received from the cell center for the D2DRs. Finally, in Ref. [16] studies a resource allocation technique that considers the interference and power optimization. However, in this case, there was a high probability for heavy collisions to occur between two links as a result of the random allocation of frequency resources regardless of the presence of a cellular link and a D2D link. Therefore, in this paper, we consider resource allocation and power management methods that reduce the interference of the cellular link and D2D link, such as the Partial Co-channel based Overlap Resource Power Control (PC.OVER) [17], and that use the frequency reuse schemes, such as the Fractional Frequency Reuse based Overlap Resource Power Control (FFR.OVER) [18], to improve the performance at the cell-edge of cellular links and for the D2D link to respond to a strong signal at the cell center.

Several schemes for inter-cell interference mitigation have been considered in orthogonal frequency division multiple access (OFDMA) networks, such as fractional frequency reuse (FFR) [19] and soft frequency reuse (SFR) [20]. Partial reuse implements different reuse factors for the cell center and the cell edge, and thus, partial reuse schemes can achieve a much higher network capacity relative to traditional frequency reuse schemes and can simultaneously reduce the inter-cell interference relative to a frequency reuse factor (FRF) of 1. However, since the cell edges use a higher reuse factor, the spectral efficiency of the cell edge may be significantly degraded relative to that at the cell center.

Ref. [21] proposed a resource allocation scheme for D2D networks with an FFR system. The scheme proposed in Ref. [22] uses four resource groups to reduce the interference of mUEs and D2D links. However, the proposed scheme uses only FFR-based frequency planning for mUEs and D2D links in the inner and outer zones, respectively. In addition, the strength of the D2DS Tx power $\left(P_{D 2 D S}\right)$ and the performance for mUEs and D2D links in inner and outer zones has not been analyzed. To solve this problem, we propose a Fractional Frequency Reuse based Adaptive Power Control (FFR.APC) Scheme for OFDMA and time division duplex (TDD) in an LTE-Advanced D2D network. In this scheme, we control and optimize the D2DR transmit power according to the user requirements in order to mitigate the interference.

The main contributions of this paper are summarized as follows:

- We propose a resource allocation problem and power control scheme to minimize the outage probability and maximize the overall network throughput by limiting the cross-tier interference at mBS below a predefined threshold

- We study the impact of D2D communication on the existing macrocells that are subject to different frequency planning strategies and optimize the power allocation between the mBSs and D2D links. The D2D throughput has also been improved by controlling the D2D power. To the best of our knowledge, previous studies have not set such an objective 


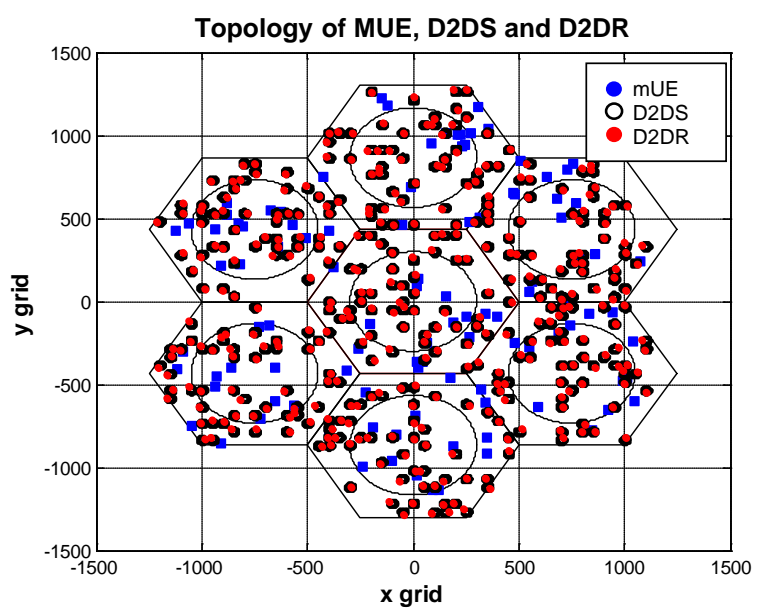

Fig. 1. System topology

- Finally, we compare the performance of the three schemes and provide recommendations as to the environment in which they are to be deployed.

Thus, the PC.OVER, FFR.OVER and FFR.APC schemes increase the overall system throughput by limiting the cross-tier interference at the mBS. Furthermore, in FFR.APC, the overall system throughput increases by limiting the cross-tier interference at the mBS below a predefined threshold level, i.e., the maximum cross-tier interference that the mBS can tolerate, by ensuring the optimization of the D2DRs power allocation for each power adjustment phase.

The results of the simulation indicate there is a significant increase in the overall system and a decrease in the outage probability.

\section{Related Work}

\subsection{Network and Channel Model}

The network consists of $N$ macrocells where each macrocell occupies a hexagonal region with a radius $R$. We assume that each mBS is located at the center of the cell, and inside of each cell site, macrocell users are assumed to be randomly distributed and to be served by OFDMA [22]. A subchannel is randomly assigned to one macro user.

The macrocell is overlaid with D2D users. Each D2DR accesses a random subset of the shared sub channels that are independent of the other D2DRs whereby each sub channel is accessed with equal probability [23]. The D2DRs are separated from their corresponding D2DSs with a distance of $q$, where $q$ is a uniform random variable within $[1,20] \mathrm{m}$.

The channel model is assumed to consist of a deterministic distance-dependent path-loss component and a random distance-independent component [24]. We assume that the signal power received, $P_{S}$ at mUE and D2DR from the mBS and D2DS can be expressed as

$$
P_{s}=P_{t} \phi_{s, t} r_{s, t}^{-\alpha},
$$

where $P_{t}$ is the transmit power of the mBS and D2DS. The distance-independent channel gain $\phi_{s, t}$ represents the lognormal $(L N)$ fading with a mean of $0 \mathrm{~dB}$ and a standard deviation of $\sigma_{s, t}$, 
where shadow fading is usually characterized in terms of its $\mathrm{dB}$ spread as $\sigma_{s, t}=\frac{\ln 10}{10} \sigma_{s, t, d B} \cdot r_{s, t}$ is the distance between the sender or the transmitter and receiver, and $\alpha$ is the path-loss exponent.

\subsection{Signal Power}

Let's assume that the central mBS, is placed at the origin and transmits to its desired user, $z$ with transmit power, $P_{z}$. Equation (1) indicates that the signal power received at the mUE can be expressed as

$$
S_{z}=P_{z} \phi_{z, z_{0}} r_{z, z_{0}}^{-\alpha},
$$

where $\phi_{z, z_{0}}$ is distributed according to $\operatorname{LN}\left(0, \sigma_{z, z_{0}}^{2}\right)$.

The mUE experiences interference from $N-1$ neighboring mBSs, $m B S_{k}$. The aggregate macrocell interference for mUE is expressed as

$$
I_{z, z}=\sum_{k=1}^{N-1} P_{z} \phi_{z, z_{k}} r_{z, z_{k}}^{-\alpha}
$$

In Eq. (3), the random variable $\phi_{z, z_{k}}$ is assumed to be distributed according to $\operatorname{LN}\left(\sigma_{z, z_{k}}^{2}\right)$.

The mUE also experiences interference from M D2DSs, and the aggregate D2DSs interference for the mUE can be expressed as

$$
I_{z, D}=\sum_{a=1}^{M-1} P_{D} \phi_{z, D_{a}} r_{z, D_{a}}^{-\alpha}
$$

where $P_{D}$ is the transmission power of D2DS, $r_{z, D_{a}}$ represents the distance between D2DS and the mUE $z$, and the random variable $\phi_{z, D_{a}}$ is distributed according to $L N\left(0, \sigma_{z, D_{a}}^{2}\right)$.

The D2DS in $\mathrm{mBS}_{1}$ communicates with D2DR at a power of $P_{D}$ using the same channel as mUE $z$. The received signal power at the D2DR is expressed as

$$
S_{D}=P_{D} \phi_{D, D_{0}} r_{D, D_{0}}^{-\alpha}
$$

where $\phi_{D, D_{0}}$ is the distribution according to $L N\left(0, \sigma_{D, D_{0}}^{2}\right)$. The received interference from the co-channel mBSs is expressed as

$$
I_{D, z}=\sum_{h=0}^{N-1} P_{z} \phi_{D, z_{h}} r_{D, z_{h}}^{-\alpha}
$$

where $\phi_{D, z_{h}}$ is distributed as $\operatorname{LN}\left(0, \sigma_{D, z}^{2}\right)$. The aggregate D2DS interference for D2DR is given as

$$
I_{D, D}=\sum_{a=1}^{M-1} P_{D} \phi_{D, D_{a}} r_{D, D_{a}}^{-\alpha}
$$

where $\phi_{D, D_{a}}$ is the distribution according to $\operatorname{LN}\left(0, \sigma_{D, D}^{2}\right)$. 


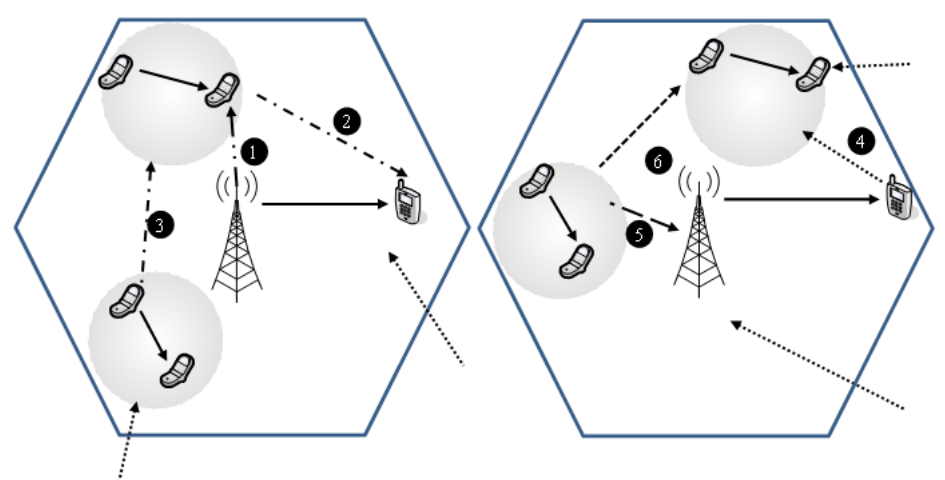

(a) Downlink

(b) Uplink

\begin{tabular}{|c|c|c|c|}
\hline Index & $\begin{array}{l}\text { Interference } \\
\text { Scenario }\end{array}$ & $\begin{array}{l}\text { Transmission } \\
\text { mode of Macro }\end{array}$ & Symbol \\
\hline 1 & $\mathrm{mBS} \rightarrow \mathrm{D} 2 \mathrm{DR}$ & Down link & $-\cdots \rightarrow$ \\
\hline 2 & $\mathrm{D} 2 \mathrm{DS} \rightarrow \mathrm{mUE}$ & Down link & $---\rightarrow$ \\
\hline 3 & D2DS $\rightarrow$ D2DR & Down link & $\cdot-\cdots \rightarrow$ \\
\hline 4 & $\mathrm{mUE} \rightarrow \mathrm{D} 2 \mathrm{DS}$ & Up link & 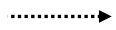 \\
\hline 5 & $\mathrm{D} 2 \mathrm{DR} \rightarrow \mathrm{mBS}$ & Up link & $--\rightarrow$ \\
\hline 6 & $\mathrm{D} 2 \mathrm{DR} \rightarrow \mathrm{D} 2 \mathrm{DS}$ & Up link & $-----\rightarrow$ \\
\hline
\end{tabular}

$\begin{array}{ll}\longrightarrow & \text { Interference } \\ \longrightarrow & \text { Signal } \\ \text { (4) } & \text { mBS } \\ 8 & \text { D2DR } \\ 8 & \text { D2DS }\end{array}$

Fig. 2. Interference scenarios in D2D networks

\subsection{Interference Scenarios in D2D Networks}

As shown in Fig. 2, we consider two types of interference that occur in a two-tier (Inter-tier and Intra-tier) D2D network architecture. Inter-tier interference occurs among network elements that belong to the same tier in the network, and in the case of a D2D network, the inter-tier interference occurs between neighboring D2D links. On the other hand, intra-tier interference occurs among network elements that belong to different tiers of the network, i.e., interference between the D2D links and macrocells.

The D2D links are deployed over the existing macrocell network and share the same frequency spectrum as the macrocells. Due to the spectral scarcity, the D2D links and the macrocells have to partially or totally reuse the total allocated frequency band, which leads to inter-tier or co-channel interference. At the same time, in order to guarantee the required QoS to the mUEs, the D2DRs should occupy as little bandwidth as possible, which leads to intra-tier interference. As a result, the network throughput would decrease substantially due to such inter-tier and intra-tier interference. Fig. 2 illustrates all possible interference scenarios in an OFDMA-based D2D network. If an effective interference management scheme can be adopted, then the inter-tier interference can be mitigated and the intra-tier interference can be reduced, which would improve the throughput of the overall network.

\subsection{Problem Formulation}

In FFR.OVER and FFR.APC, the outage performance is analyzed for specific user positions. In this section, the total throughput performance is investigated to evaluate each of the schemes. The throughput of the schemes employed in the proposed spectrum reuse scheme can be expressed as

$$
T=\left\{N\left(1-\eta_{D}(k)\right)+\left(1-\eta_{z}(k)\right)\right\}, 0<k \leq R,
$$




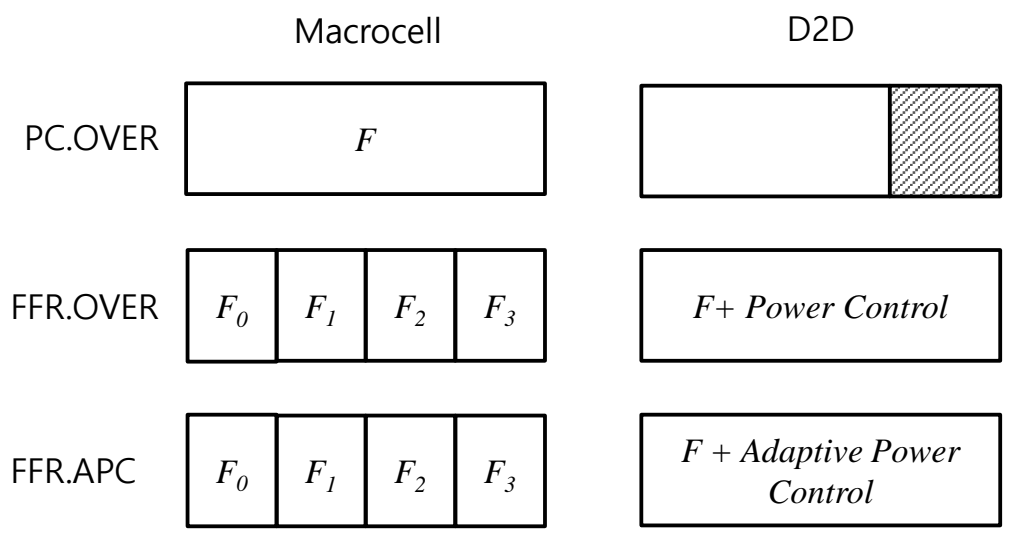

Fig. 3. Spectrum reuse Strategies

where $R$ is macrocell radius, $\eta_{D}(k)$ and $\eta_{z}(k)$ are obtained by averaging the outage probabilities of the inner and outer regions and they are respectively given by $\eta_{D}(k)=E\left[\eta_{D, \text { outer }} \mid r_{D, D_{0}}>k\right]+E\left[\eta_{D, \text { inner }} \mid r_{D, D_{0}}<k\right]$ and $\eta_{z}(k)=E\left[\eta_{z, \text { outer }} \mid r_{z, z_{0}}>k\right]+E\left[\eta_{z, \text { inner }} \mid r_{z, z_{0}}<k\right]$.

For PC.OVER, the throughput can be expressed as

$$
T=\left\{\beta N\left(1-\eta_{D}(R)\right)+\left(1-\eta_{z}(R)\right)\right\},
$$

where $\beta$ is the resource allocation ratio defined in (11).

The effective throughput depends on the outage probability in the cell. We can express our problem as optimization problem as follows

$$
\begin{array}{ll}
\text { maximize } & \multicolumn{1}{c}{T,} \\
\text { subject to } & \left(\eta_{D} \& \& \eta_{z}\right) \leq \eta \\
\text { variable } & P_{D}, P_{z}
\end{array}
$$

where $\eta_{D}$ and $\eta_{z}$ are the outage probabilities for D2D and mUE whose threshold is $\eta$.

\section{Spectrum Reuse with Power Control for D2D}

In this paper, we present and evaluate the schemes to improve the D2D performance but also limit cross-tier interference to the macrocell. In this section, the spectrum reuse and power control in the three schemes - PC.OVER, FFR.OVER and FFR.APC - are discussed in further detail.

\subsection{Spectrum Reuse Schemes}

The spectrum reuse strategies considered in this paper are shown in Fig. 3. For the PC.OVER scheme, the total available bandwidth $F$ is used by mUE in macrocell. The D2D users are allowed to use a portion of the available bandwidth from the total available bandwidth. If we define $\beta$ as the resource allocation ratio, then the bandwidth $F_{D}$ that is available for the D2D user to use at any time depends on $\beta$ and is given as

$$
F_{D}=\beta F .
$$

For the FFR.OVER and FFR.APC schemes, the total available bandwidth $F$ that is available for mUE is partitioned into four orthogonal frequency bands, namely $F_{0}, F_{1}, F_{2}$ 
and $F_{3}$, where $F=F_{0}+F_{1}+F_{2}+F_{3}$. The D2D users are allowed to use any portion of the total bandwidth available when using the power control scheme that is explained in 3.2.

\subsection{D2D Power Control Mechanism}

As shown in Fig. 3, the mUE is allowed to use the total available bandwidth $F$. The mUE transmits in any of the available RB from the 50 RBs that are available. PC.OVER assigns the minimum power to those D2DRs (more than one) that compete for the same RB with mUE. When more than one D2DRs compete for the same RB with mUE, allowing the D2DRs to transmit with their maximum power, co-channel interference (CCI) becomes severe. To avoid this and to further mitigate interference, for those RBs where more than one D2DRs compete for the same resource with mUE, the power for the competing D2DRs is reduced to their minimum power level. It is important to note that when there is no D2DR competition, we have a normal collision. Algorithm 1 shows the procedure with which the mBSs allocate the RB to D2DRs by considering power control in the D2DRs through the use of the proposed scheme.

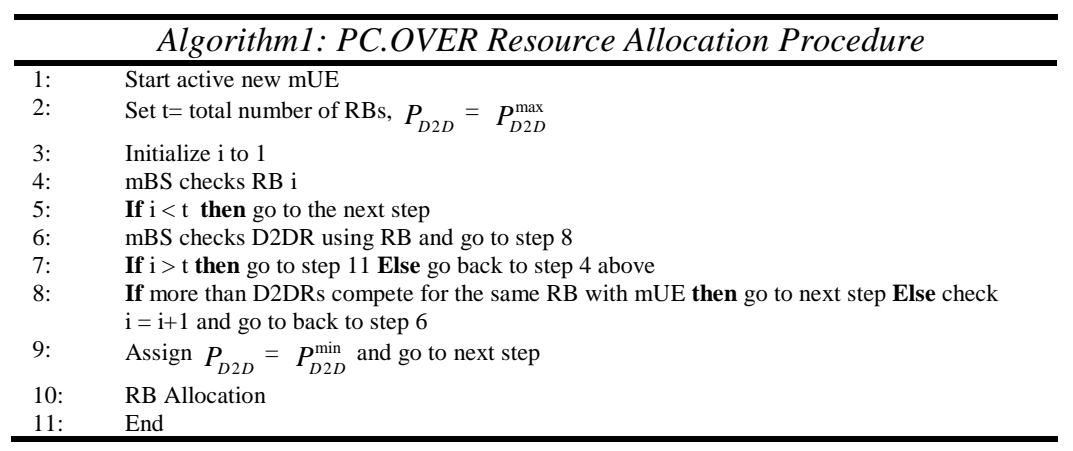

The FFR.OVER and FFR.APC schemes combine the FFR techniques and the power control mechanism. The entire frequency band is partitioned into two parts, including central and edge sub-bands. A reuse factor of one is used in the cell edge region while a reuse factor of three is deployed in the edge regions of adjacent cells to prevent Inter-cell interference and adjacent cell interference. Accordingly, the overall capacity of the system improves. The sub-bands are denoted by $F_{n}$, where $n$ is the number that corresponds to the associated sub-band. From Fig. 3, $F_{0}$ is the central sub-band and $F_{1}, F_{2}$ and $F_{3}$ are the sub-bands at the edge regions.

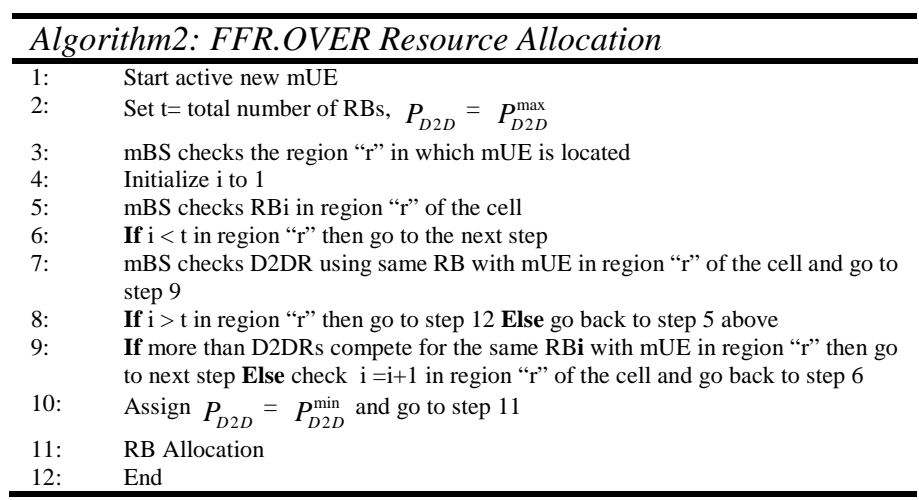




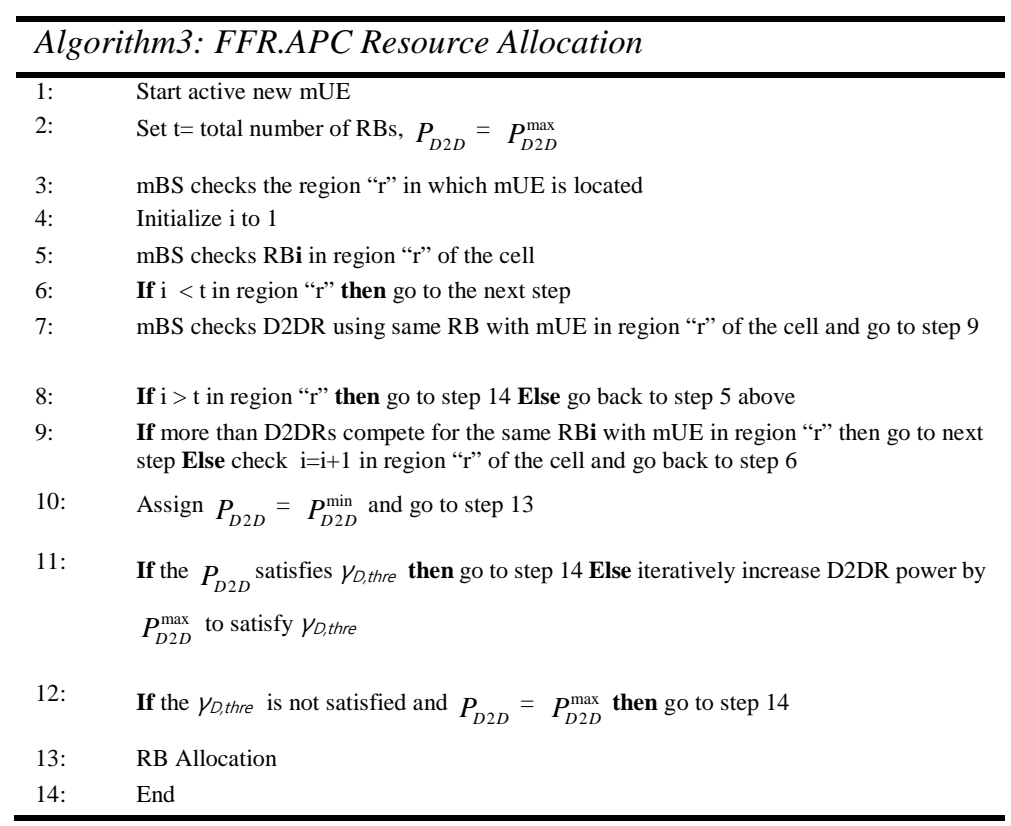

In our scheme, all users, i.e., mUEs and D2DRs, transmit data in their respective bands with uniform power and are randomly allocated over the regions. $50 \mathrm{RBs}$, i.e., $10 \mathrm{MHz}$ of bandwidth, are considered in each scheme, and the mUEs are free to transmit in any of these RBs. The mUE at the inner region will transmit using the central sub-band $F_{0}$, whereas at the edge regions it will be transmitting data using the corresponding allocated sub-band in that region, i.e., $F_{1}, F_{2}$ or $F_{3}$. For D2D communication, the D2DR can transmit using any part of the sub-band that it has been allocated. For example, it will use $F_{0}$ when it is in the center region and $F_{1}, F_{2}$ or $F_{3}$ when at the edge regions, depending on the location. This creates interference, and the interference is even further accelerated when more than one D2DRs simultaneously share the same RB with another D2DR or mUE during transmission and these D2DRs use the maximum transmission power. To prevent further interference, the FFR.OVER scheme requires D2DRs sharing the RB to be set to transmit with the minimum amount of power for D2DRs that compete for resources with the mUE. The sub-band is reused as much as possible since the transmit power of the D2DS is reduced to its minimum power. Reusing the sub-band with a considerable number of D2DRs that transmit with a low power not only greatly avoids interference but also improves the system performance. The FFR.APC scheme further improve the system performance by iteratively increasing the power of D2DR by a factor that is equivalent to its minimum power in order to meet its SINR threshold. It is important to note that no power has increased when the D2DR SINR satisfies the required SINR of the D2DR threshold. The power can be iteratively increased until the maximum D2DR power has been reached, and if the required SINR threshold is not satisfied, then the process stops. The procedures for how the mBSs allocates the RB to D2DRs as proposed for the FFR.OVER and FFR.APC schemes by considering the power control of the D2DRs can be found in the Algorithms 2 and 3, respectively. 


\section{Experimental Classification Results and Analysis}

\subsection{Signal to Interference Plus Noise Ratio (SINR)}

The outage probability for the macrocell can be obtained depending on the type of scheme that has been used and the power control that is explained for each scheme. The SINR received at the mUE can be expressed as

$$
\gamma_{z}=\frac{S_{z}}{N_{0}+I_{z, z}+I_{z, D}} .
$$

Substituting (2), (3) and (4) in (12), we rewrite (12) as

$$
\gamma_{z}=\frac{P_{z} \phi_{z, z_{0}} r_{z, z_{0}}^{-\alpha}}{N_{0}+\sum_{k=1}^{N-1} P_{z} \phi_{z, z_{k}} r_{z, z_{k}}^{-\alpha}+\sum_{a=1}^{M-1} P_{D} \phi_{z, D_{a}} r_{z, D_{a}}^{-\alpha}} .
$$

The D2D user will experience interference from the mUE and the other D2Ds. The SINR received at the D2DR can be expressed as

$$
\gamma_{D}=\frac{S_{D}}{N_{0}+I_{D, z}+I_{D, D}} .
$$

Substituting (5), (6) and (7) in (14), we rewrite (14) as

$$
\gamma_{D}=\frac{P_{D} \phi_{D, D_{0}} r_{D, D_{0}}^{-\alpha}}{N_{0}+\sum_{h=0}^{N-1} P_{z} \phi_{D, z_{h}} r_{D, z_{h}}^{-\alpha}+\sum_{a=1}^{M-1} P_{D} \phi_{D, D_{a}} r_{D, D_{a}}^{-\alpha}} .
$$

\subsection{Outage probability}

The outage probability is defined as the probability that the SIR of the mUE or D2DR falls below the respective thresholds. Accordingly, the outage probability for the mUE and the D2DR can be respectively expressed as

$$
\begin{gathered}
\eta_{z}=\operatorname{Pr}\left(\gamma_{z}<\gamma_{z, \text { thre }}\right), \\
\eta_{D}=\operatorname{Pr}\left(\gamma_{D}<\gamma_{D, \text { thre }}\right),
\end{gathered}
$$

where $\gamma_{z, \text { thre }}$ and $\gamma_{D, \text { thre }}$ are the mUE and D2DR thresholds, respectively.

\section{Performance Analysis}

We conducted a Monte Carlo simulation to investigate and evaluate the DL performance of the three frequency planning and power management schemes in terms of the system throughput and outage probability of the mUEs and D2DRs for the D2D networks. We performed 10,000 independent simulations and evaluated the system performance according to the number of D2DSs in the analysis. For the three schemes, we assume that the mBS and D2DSs allocate only one RB for each mUE and D2DR, respectively. The mBS does not allocate the same RBs to the mUEs in the same cell, but the D2DSs allocate an RB randomly in the allocated channel groups for each D2DR. The detailed explanation and description for the performance measurements is given in Ref. [25]. The other simulation parameters for all schemes are given in Table $\mathbf{1}$. 
The system performance of the proposed scheme is evaluated in terms of the system throughput and the outage, and the results are compared for the three schemes in order to observe which one outperforms the others.

Table 1. System parameters

\begin{tabular}{|c|c|c|c|}
\hline \multirow[t]{2}{*}{ Parameters } & \multicolumn{3}{|c|}{ Values } \\
\hline & PC.OVER & FFR.OVER & FFR.APC \\
\hline Carrier Frequency & \multicolumn{3}{|c|}{$2 \mathrm{GHz}$} \\
\hline mBS Cell Layout & \multicolumn{3}{|c|}{ Hexagonal } \\
\hline Bandwidth for DL & \multicolumn{3}{|c|}{$10 \mathrm{MHz}$} \\
\hline Number of sites & \multicolumn{3}{|c|}{7 cells } \\
\hline $\mathrm{mBS} / \mathrm{D} 2 \mathrm{D}$ radius & \multicolumn{3}{|c|}{$866 \mathrm{~m} / 20 \mathrm{~m}$} \\
\hline \multirow{2}{*}{$\begin{array}{l}\text { mBS Tx power } \\
\left(P_{\text {mBS }}\right)\end{array}$} & \multirow{2}{*}{\multicolumn{2}{|c|}{$41.7 \mathrm{dBm}(15 \mathrm{~W})$}} & $\begin{array}{c}\text { Inner zone: } \\
\text { 41.17dBm (15W) }\end{array}$ \\
\hline & & & $\begin{array}{c}\text { Outer zone: } \\
43.42 \mathrm{dBm}(22 \mathrm{~W})\end{array}$ \\
\hline \multirow{2}{*}{$\begin{array}{c}\text { D2DS Tx power } \\
\left(P_{\text {D205 }}\right)\end{array}$} & \multicolumn{3}{|c|}{ Minimum power $=8 \mathrm{dBm}(6.3 \mathrm{~mW})$} \\
\hline & \multicolumn{3}{|c|}{ Maximum power $=24 \mathrm{dBm}(251 \mathrm{~mW})$} \\
\hline Number of D2D pairs & \multicolumn{3}{|c|}{200} \\
\hline Number mUE & \multicolumn{3}{|c|}{30} \\
\hline Antenna pattern & \multicolumn{3}{|c|}{ Omni directional } \\
\hline Traffic mode & \multicolumn{3}{|c|}{ Full buffer } \\
\hline SIR threshold, $\eta$ & \multicolumn{3}{|c|}{$-6 \mathrm{~dB}$} \\
\hline $\begin{array}{l}\text { Noise power density } \\
\left(\mathrm{N}_{0}\right)\end{array}$ & \multicolumn{3}{|c|}{$-174 \mathrm{dBm} / \mathrm{Hz}$} \\
\hline
\end{tabular}

Fig. 4 shows the results of the system throughput for mUE and D2D for each of the schemes discussed in our paper. As can be seen from Fig. 4(a), the spatially averaged macrocell throughput that is achievable for the network system is near that of an optimal network when our throughput based design is used with PC.OVER, FFR.OVER, or FFR.APC. The macrocell throughput that is achievable for the optimized design decreases as the number of interfering D2Ds increases because the CCI imposed by the high density D2DS is stronger than that of the low density systems. The system throughput is higher for FFR.APC compared17.18 Mbps, and 17.57 Mbps, respectively. Again, if we consider the system to support 200 D2Ds and a power of $0.2463 \mathrm{~W}$, it can be seen that FFR.APC provides a throughput of 15.12 Mbps while PC.OVER and FFR.OVER provide 14.16 Mbps and 14.77 Mbps, respectively. The decrease in the throughput is due to the increase in interference following the deployment of a greater number of D2Ds and also from neighboring mUEs. Table 2 provides different values for the system throughput at different D2D power levels for the mUE.

Fig. 4 (b) shows the results of the system throughput for D2D. As can be seen from Fig. 4(b), the system throughput is higher for FFR.APC than for the other schemes for the same value of D2D power as for its mUE counterpart. It can also be observed that, when the D2D power increases, the D2D throughput increases. For example, considering 200 D2Ds, the system throughput value for PC.OVER, FFR.OVER and FFR.APC are approximately 343 Mbps, $380 \mathrm{Mbps}$ and $383 \mathrm{Mbps}$, respectively for a D2D power of $0.24633 \mathrm{~W}$, and it is 198 Mbps, $308 \mathrm{Mbs}$ and $343 \mathrm{Mbps}$ for a D2D power of $0.0063 \mathrm{~W}$ respectively. to other schemes with the same D2D power. For example, if we consider 100 D2Ds and a power of 0.0063W, the throughput for PC.OVER, FFR.OVER and FFR.APC are 16.53 Mbps,

However, the general trend for all schemes is that the system throughput increases linearly as the number of D2D increases, and in both cases, FFR.APC shows a better system throughput. Table 3 provides the different values for the system throughput at different D2D power levels 
for D2D.
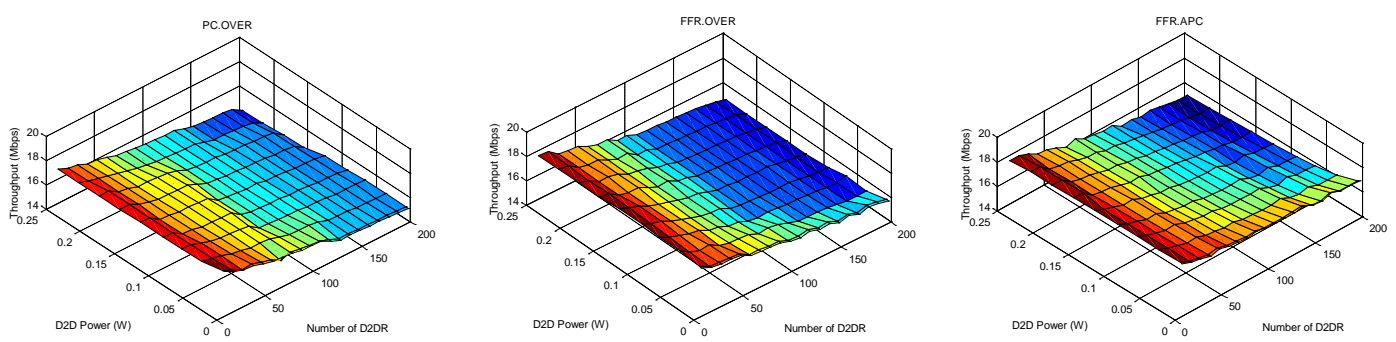

(a) System Throuhput for mUE
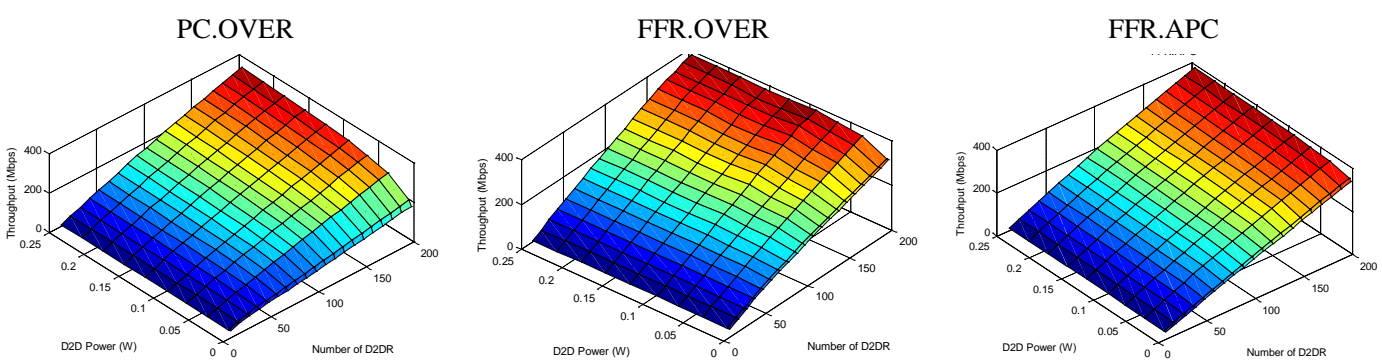

(b) System Throuhput for D2D

Fig. 4. The system throughput for (a) mUE and (b) D2D

Fig. 5 shows the results of the outage probability for mUE and D2D for each scheme that is discussed in our paper. Fig. 5(a) clearly shows that, as expected, the spatially averaged outage probability increases as the number of D2DRs increases within all schemes that are considered. Specifically, the outage probability of PC.OVER is significantly higher than that for both FFR.OVER and FFR.APC due to the fact that the CCI imposed on the mUE for PC.OVER is significantly higher than that of the other two schemes. Furthermore, the outage probability for FFR.APC is lower than that for FFR.OVER, which has been achieved at the cost of a decrease in the throughput.

Table 2. Total System Throughput for mUE (Mbps)

\begin{tabular}{|c|l|l|l|l|l|l|l|l|l|}
\hline \multirow{2}{*}{$\begin{array}{c}\text { Number of } \\
\text { D2D }\end{array}$} & \multirow{2}{*}{ Scheme } & \multicolumn{9}{|c|}{ D2D Pow (W) } \\
\cline { 2 - 10 } & & $\mathbf{0 . 0 0 6 3}$ & $\mathbf{0 . 0 6 6 3}$ & $\mathbf{0 . 0 9 6 3}$ & $\mathbf{0 . 1 2 6 3}$ & $\mathbf{0 . 1 5 6 3}$ & $\mathbf{0 . 1 8 6 3}$ & $\mathbf{0 . 2 1 6 3}$ & $\mathbf{0 . 2 4 6 3}$ \\
\hline \multirow{3}{*}{50} & PC.OVER & 16.53 & 16.34 & 16.28 & 16.25 & 16.20 & 16.18 & 16.15 & 16.13 \\
\cline { 2 - 10 } & FR.OVER & 17.18 & 16.85 & 16.74 & 16.68 & 16.64 & 16.94 & 16.95 & 16.94 \\
\cline { 2 - 10 } & FFR.APC & 17.57 & 17.34 & 17.28 & 17.24 & 17.16 & 17.12 & 17.09 & 17.00 \\
\hline \multirow{3}{*}{100} & PC.OVER & 15.83 & 15.40 & 15.40 & 15.40 & 15.40 & 15.40 & 15.40 & 15.40 \\
\cline { 2 - 10 } & FFR.OVER & 16.30 & 15.58 & 15.63 & 15.62 & 15.51 & 15.50 & 15.48 & 15.45 \\
\cline { 2 - 10 } & FFR.APC & 17.10 & 16.55 & 16.41 & 16.30 & 16.42 & 16.34 & 16.28 & 16.15 \\
\hline \multirow{3}{*}{150} & PC.OVER & 15.17 & 15.16 & 15.15 & 15.15 & 15.14 & 15.13 & 14.83 & 14.80 \\
\cline { 2 - 10 } & FFR.OVER & 15.84 & 15.37 & 15.35 & 15.35 & 15.32 & 15.32 & 15.32 & 15.32 \\
\cline { 2 - 10 } & FFR.APC & 16.84 & 16.09 & 15.91 & 15.77 & 15.77 & 15.66 & 15.57 & 15.46 \\
\hline \multirow{3}{*}{200} & PC.OVER & 15.00 & 15.02 & 14.88 & 14.83 & 14.37 & 14.33 & 14.20 & 14.16 \\
\cline { 2 - 10 } & FFR.OVER & 15.62 & 15.27 & 14.96 & 14.86 & 14.77 & 14.77 & 14.77 & 14.77 \\
\cline { 2 - 10 } & FFR.APC & 16.82 & 15.79 & 15.58 & 15.42 & 15.36 & 15.24 & 15.15 & 15.12 \\
\hline
\end{tabular}


Table 3. Total System Throughput for D2DR (Mbps)

\begin{tabular}{|c|l|c|c|c|c|c|c|c|c|}
\hline \multirow{2}{*}{$\begin{array}{c}\text { Number of } \\
\text { D2D }\end{array}$} & \multirow{2}{*}{ Scheme } & \multicolumn{9}{|c|}{ D2D Power $(\mathbf{W})$} \\
\cline { 3 - 9 } & & $\mathbf{0 . 0 0 6 3}$ & $\mathbf{0 . 0 6 6 3}$ & $\mathbf{0 . 0 9 6 3}$ & $\mathbf{0 . 1 2 6 3}$ & $\mathbf{0 . 1 5 6 3}$ & $\mathbf{0 . 1 8 6 3}$ & $\mathbf{0 . 2 1 6 3}$ & $\mathbf{0 . 2 4 6 3}$ \\
\hline \multirow{3}{*}{50} & PC.OVER & 68.15 & 88.01 & 91.19 & 93.48 & 94.98 & 96.43 & 97.66 & 99.26 \\
\cline { 2 - 10 } & FFR.OVER & 101.07 & 106.06 & 108.05 & 106.87 & 106.87 & 108.02 & 107.43 & 107.43 \\
\cline { 2 - 10 } & FFR.APC & 102.30 & 107.00 & 108.14 & 108.89 & 109.57 & 109.57 & 109.94 & 110.44 \\
\hline \multirow{3}{*}{100} & PC.OVER & 109.12 & 160.14 & 168.13 & 173.85 & 178.30 & 181.88 & 184.89 & 187.25 \\
\cline { 2 - 10 } & FFR.OVER & 177.14 & 200.90 & 210.22 & 205.50 & 205.50 & 210.37 & 209.86 & 209.86 \\
\cline { 2 - 10 } & FFR.APC & 198.04 & 216.96 & 220.03 & 221.87 & 223.86 & 224.97 & 225.30 & 226.04 \\
\hline \multirow{3}{*}{150} & PC.OVER & 153.15 & 228.61 & 240.13 & 248.31 & 255.10 & 260.17 & 264.41 & 268.01 \\
\cline { 2 - 10 } & FFR.OVER & 244.04 & 289.72 & 307.25 & 298.66 & 298.66 & 307.04 & 307.04 & 307.04 \\
\cline { 2 - 10 } & FFR.APC & 263.45 & 298.13 & 304.09 & 307.89 & 310.18 & 311.90 & 313.24 & 314.52 \\
\hline \multirow{3}{*}{200} & PC.OVER & 197.64 & 293.97 & 308.37 & 318.51 & 324.79 & 331.00 & 336.17 & 342.89 \\
\cline { 2 - 9 } & FF.OVER & 307.89 & 374.99 & 382.69 & 377.20 & 377.20 & 380.24 & 380.24 & 380.24 \\
\cline { 2 - 9 } & FFR.APC & 343.18 & 388.90 & 384.76 & 380.18 & 378.55 & 380.99 & 381.60 & 382.66 \\
\hline
\end{tabular}
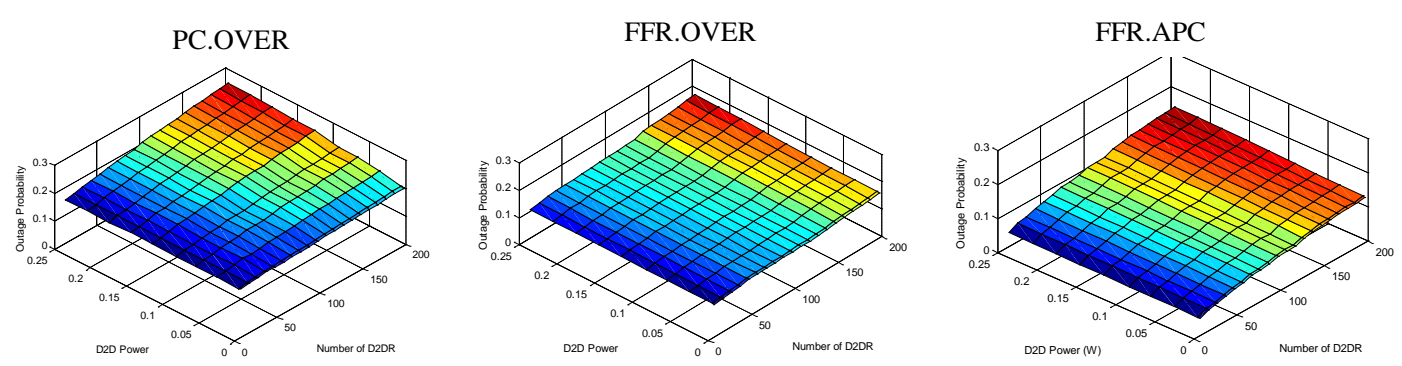

(a) Outage probability for mUE
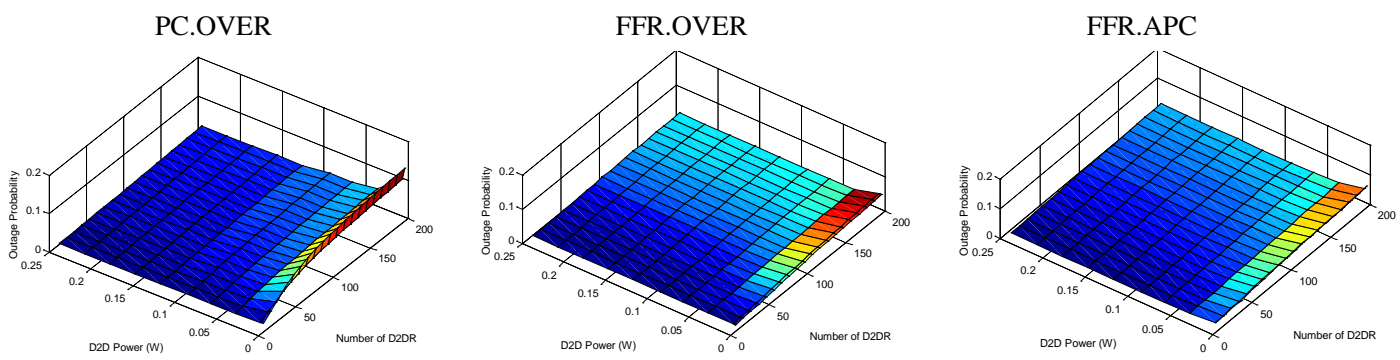

(b) Outage probability for D2D

Fig. 5. The outage probability for (a) mUE and (b) D2D

If we design our system with a 0.1 outage probability requirement, we can recommend FFR.APC with 10 D2Ds to 100 D2Ds. For example, if we consider 50 D2Ds and a power of $0.0063 \mathrm{~W}$, the outage probabilities for PC.OVER, FFR.OVER and FFR.APC are 0.1745, 0.1238 , and $\mathbf{0 . 0 7 3 1}$ respectively. Again, if we consider the system to support 100 D2Ds and a power of $0.2463 \mathrm{~W}$, FFR.APC can be seen to have a low outage probability of $\mathbf{0 . 1 0 9 9}$ while PC.OVER and FFR.OVER have outage probabilities of 0.2122 and 0.1378 , respectively. The increase in the outage probability is a result of interference that increases following the deployment of a greater number of D2Ds while the interference increases from neighboring mUEs. Table 4 provides different values of the total system throughput at different D2D power values for mUE.

Fig. 5(b) shows the results of the outage probability for D2D, as can be seen from Fig. 5(b), the outage probability is higher for PC.OVER relative to other schemes for the same value of D2D power as for its counterpart mUE. When the D2D power increases, the D2D outage probability can be observed to increase since the interference also increases. For example, considering 200 D2Ds, the outage probabilities for PC.OVER, FFR.OVER and FFR.APC are 
approximately $0.0979,0.0462$ and 0.0249 , respectively, for a D2D power of $0.0063 \mathrm{~W}$, and these are $0.0257,0.0223$ and 0.0183 for a D2D power of $0.2463 \mathrm{~W}$, respectively. However, the general trend for all schemes is that the system outage probability increases linearly as the number of D2D increases. In both cases, FFR.APC shows a low outage probability.

Table 4. Outage probability for mUE ( $\eta_{z} \leq 0.1$ as see in highlight value)

\begin{tabular}{|c|c|c|c|c|c|c|c|c|c|}
\hline \multirow{2}{*}{$\begin{array}{c}\text { Number of } \\
\text { D2D }\end{array}$} & \multirow{2}{*}{ Scheme } & \multicolumn{8}{|c|}{ D2D Power (W) } \\
\hline & & 0.0063 & 0.0663 & 0.0963 & 0.1263 & 0.1563 & 0.1863 & 0.2163 & 0.2463 \\
\hline \multirow{3}{*}{50} & PC.OVER & 0.1745 & 0.1800 & 0.1800 & 0.1846 & 0.1846 & 0.1845 & 0.1865 & 0.1862 \\
\hline & FFR.OVER & 0.1238 & 0.1244 & 0.1252 & 0.1269 & 0.1279 & 0.1287 & 0.1297 & 0.1299 \\
\hline & FFR.APC & 0.0731 & 0.0760 & 0.0772 & 0.0783 & 0.0793 & 0.0803 & 0.0812 & 0.0820 \\
\hline \multirow{3}{*}{60} & PC.OVER & 0.1760 & 0.1834 & 0.1834 & 0.1876 & 0.1886 & 0.1895 & 0.1910 & 0.1916 \\
\hline & FFR.OVER & 0.1248 & 0.1253 & 0.1256 & 0.1279 & 0.1283 & 0.1296 & 0.1306 & 0.1307 \\
\hline & FFR.APC & 0.0760 & 0.0792 & 0.0805 & 0.0819 & 0.0827 & 0.0838 & 0.0849 & 0.0859 \\
\hline \multirow{3}{*}{70} & PC.OVER & 0.1796 & 0.1829 & 0.1889 & 0.1941 & 0.1951 & 0.1966 & 0.1968 & 0.1992 \\
\hline & FFR.OVER & 0.1257 & 0.1265 & 0.1275 & 0.1282 & 0.1290 & 0.1302 & 0.1312 & 0.1324 \\
\hline & FFR.APC & 0.0840 & 0.0874 & 0.0887 & 0.0901 & 0.0914 & 0.0926 & 0.0937 & 0.0948 \\
\hline \multirow{3}{*}{80} & PC.OVER & 0.1789 & 0.1898 & 0.1900 & 0.1962 & 0.1972 & 0.1991 & 0.1998 & 0.2022 \\
\hline & FFR.OVER & 0.1265 & 0.1284 & 0.1290 & 0.1302 & 0.1312 & 0.1320 & 0.1330 & 0.1345 \\
\hline & FFR.APC & 0.0842 & 0.0878 & 0.0892 & 0.0904 & 0.0915 & 0.0928 & 0.0939 & 0.0951 \\
\hline \multirow{3}{*}{90} & PC.OVER & 0.1782 & 0.1905 & 0.1905 & 0.2013 & 0.2023 & 0.2033 & 0.2045 & 0.2049 \\
\hline & FFR.OVER & 0.1280 & 0.1307 & 0.1315 & 0.1327 & 0.1337 & 0.1346 & 0.1356 & 0.1368 \\
\hline & FFR.APC & 0.0891 & 0.0929 & 0.0944 & 0.0959 & 0.0974 & 0.0990 & 0.1002 & 0.1014 \\
\hline \multirow{3}{*}{100} & PC.OVER & 0.1823 & 0.1966 & 0.1976 & 0.2065 & 0.2075 & 0.2081 & 0.2108 & 0.2122 \\
\hline & FFR.OVER & 0.1298 & 0.1315 & 0.1329 & 0.1338 & 0.1348 & 0.1351 & 0.1361 & 0.1378 \\
\hline & FFR.APC & 0.0969 & 0.1008 & 0.1025 & 0.1040 & 0.1056 & 0.1072 & 0.1085 & 0.1099 \\
\hline
\end{tabular}

\section{Conclusion}

In this paper, we discussed, analyzed and evaluated three different interference management schemes in D2D networks that make use of PC.OVER, FFR.OVER, and FFR.APC schemes in terms of the outage probabilities and the average throughput that could be obtained. Spectrum reuse schemes with power control for the PC.OVER, FFR.OVER, and FFR.APC schemes have been investigated to minimize the outage probability and maximize the spatially averaged throughput. The results of the simulation are compared among three schemes in order to determine which outperforms the others. The results of the simulation show that the three schemes outperform a conventional scheme. In addition, the results also showed that there is a tradeoff between the outage probability and the average effective throughput. In this paper, we recommend deploying the FFR.APC scheme since it showed a significant increase in the average effective throughput and low probability of an outage when compared to the PC.OVER and FFR.OVER schemes.

\section{References}

[1] Q. Li, R. Q. Hu, Y. Qian, and G. Wu, "Cooperative communications for wireless networks: techniques and applications in LTE-advanced systems,” IEEE Wireless Commun., vol. 19, no. 2, pp. 22-29, Apr. 2012. Article (CrossRef Link)

[2] 3GPP TR 22.803 V12.2.0, “Feasibility study for proximity service (ProSe),” 2013.

Article (CrossRef Link)

[3] L. Lei, Z. Zhong, C. Lin, and X. Shen, “Operator controlled device-to-device communications in LTE-Advanced networks,” IEEE Wireless Commun., vol. 19, no. 3, pp. 96-104, Jun. 2012. Article (CrossRef Link)

[4] Y. Zou, Y. Yao, B. Zheng, "Cognitive Transmissions with Multiple Relays in Cognitive Radio Networks,” IEEE Trans. Wireless Commun., vol. 10, no. 2, Feb. 2011. Article (CrossRef Link) 
[5] P. Janis, C.-H. Yu, K. Doppler, C. B. Ribeiro, C. Witjing, K. Hugl, O. Tirkkonen, and V. Koivunen, “Device-to-Device Communication Underlaying Cellular Communications Systems,” Inter. J. of Commun., Network and System Sciences, vol. 2, no. 3, pp. 169-178, Jun. 2009.

Article (CrossRef Link)

[6] K. Zhu, E. Hossain, "Joint Mode Selection and Spectrum Partitioning for Device-to-Device Communication: A Dynamic Stackelberg Game,” IEEE Trans. Wireless Commun., vol. 14, no. 3, Mar. 2015. Article (CrossRef Link)

[7] L. Lei; X. Shen; M. Dohler, C. Lin; Z. Zhong, "Queuing Models With Applications to Mode Selection in Device-to-Device Communications Underlaying Cellular Networks,” IEEE Trans. Wireless Commun., vol. 13, no. 12, Dec. 2014. Article (CrossRef Link)

[8] G. Ding, J. Wang, Q. Wu, Y. Yao, F. Song, T. A. Tsiftsis, "Cellular-Base-Station Assisted Device-to-Device Communications in TV White Space,” IEEE J. on Selected Areas in Commun., vol. PP, no. 99, May. 2015. Article (CrossRef Link)

[9] X. Xiao, X. Tao and J. Lu, “A Qos-aware power optimization scheme in OFDMA systems with integrated device-to-device (D2D) communications,” IEEE Veh. Tech. Conf., pp. 1-5, Sep. 2011. Article (CrossRef Link)

[10] S.Y. Kim, C.H. Lim, C.H. Cho, "Performance Analysis of a Dense Device to Device Network," KSII Trans. Internet and Information Systems, vol. 8, no. 9, Sep. 2014. Article (CrossRef Link)

[11] S. Bu, F.R. Yu, H. Yanikomeroglu, "Interference-Aware Energy-Efficient Resource Allocation for OFDMA-Based Heterogeneous Networks With Incomplete Channel State Information,” IEEE Trans. Veh. Tech., vol. 64, no. 3, Mar. 2015. Article (CrossRef Link)

[12] X. Wu, S. Tavildar, S. Shakkottai, T. Richardson, J. Li, R. Laroia, and A. Jovicic, "FlashLinQ: A Synchronous Distributed Scheduler for Peer-to-Peer Ad Hoc Networks,” IEEE/ACM Trans. Networking, vol. 21, no.4, pp. 1215-1228, Aug. 2013. Article (CrossRef Link)

[13] N. Naderializadeh and A. S. Avestimehr, "ITLinQ: A New Approach for Spectrum Sharing in Device-to-Device Communication Systems,” IEEE J. Sel. Commun., vol. 32, no. 6, pp. 1139-1151, Jun. 2014. Article (CrossRef Link)

[14] D. Yoon, K. Cho, J. Lee, "Bit Error Probability of Mary Quadrature Amplitude Modulation," IEEE Veh. Tech. Conf., vol.5, pp. 2422-2427, Sep. 2000. Article (CrossRef Link)

[15] P. Janis, V. Koivunen, C. Ribeiro, J. Korhonen, K. Doppler and K. Hugl, "Interference-Aware Resource Allocation for Device-to-Device Radio Underlaying Cellular Networks,” IEEE Veh. Tech. Conf., pp. 1-5, Apr. 2009. Article (CrossRef Link)

[16] C. Yu, O. Tirkkonen, K. Doppler, and C. Ribeiro, "Power Optimization of device-to-device communication underlaying cellular communication,” IEEE ICC, pp. 1-5, Jun. 2009. Article (CrossRef Link)

[17] S. Chhorn, T.S. Kim, M.H. Mohsini, S.Y. Kim, C.H. Cho, "Partial Co-channel based Overlap Resource Power Control for Interference Mitigation in an LTE-Advanced Network with Device-to-Device Communication,” CTRQ 2013, pp. 52-57, Apr. 2013 Article (CrossRef Link)

[18] S. Chhorn, S.O. Seo, S.Y. Kim, K.H. Lee, C.H. Cho, "Fractional Frequency Reuse (FFR) based Overlap Resource Power Control for Interference Mitigation in an LTE-Advanced Network with Device-to-Device Communication,” CUTE 2014, vol. 330, pp. 967-973, Dec. 2014. Article (CrossRef Link)

[19] WiMAX Forum, “Mobile WiMAX part 1: A technical overview and performance evaluation,” Jun. 2006. Article (CrossRef Link)

[20] R1-050841, Huawei, "Further Analysis of Soft Frequency Reuse Scheme," 3GPP TSG RAN WG1\#42, Aug. 29- Sep. 2 2005. Article (CrossRef Link)

[21] H. Zhu, J. Wang, "Device-to-device communication in cellular networks with fractional frequency reuse,” IEEE ICC, pp. 5503-5507, Jun. 2014. Article (CrossRef Link)

[22] C. Y. Wong, R. S. Cheng, K. B. Letaief, and R. D. Murch. "Multiuser OFDM with adaptive subcarrier, bit, and power allocation,” IEEE J. Sel. Areas Commun., vol. 17, pp. 1747-1758, Oct. 1999. Article (CrossRef Link)

[23] J. Zander and S.-L. Kim. “Radio Resource Management for Wireless Networks,” 11-50. MA: Artech House, Norwood, 2001. Article (CrossRef Link) 
[24] V. Erceg, L. J. Greenstein, S. Y. Tjandra, S. R. Parkoff, A. Gupta, B. Kulic, A. A. Julius, and R. Bianchi, “An empirically based path loss model for wireless channels in suburban environments," IEEE J. Sel. Areas Commun., vol. 17, no. 7, pp. 1205-1211, Jul. 1999. Article (CrossRef Link)

[25] T. S. Kim, K. H. Lee, S. Ryu and C. H. Cho, "Resource Allocation and Power Control Scheme for Interference Avoidance in an LTE-Advanced Cellular Networks with Device-to-Device Communication,” Inter. J. of Control and Automation, vol. 6, Feb. 2013. Article (CrossRef Link)
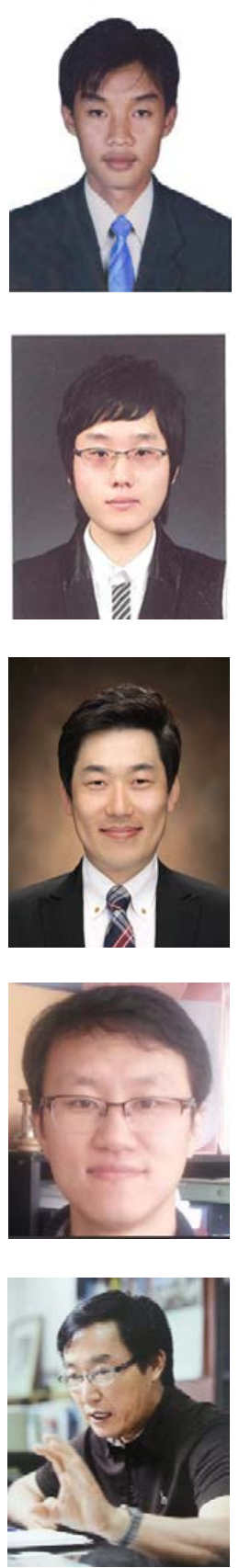

Sok Chhorn received the B.S. degree in computer science from Norton University, Cambodia in 2005, and the M.S. degree in Computer and Information Science from Korea University, Korea in 2011. He is currently pursuing the Ph.D. degree with the Department of Computer and Information Science, Korea University, Korea. His research interests include radio resource management, interference management, small cell, and device-to-device communication.

Seok-Ho Yoon received the B.S and M.S degrees in Computer and Information Science in 2009 and 2011, respectively, from Korea University, Korea, where he is currently working toward the Ph.D. degree in the Laboratory for Data Communication Networks, Korea University. He has participated in various research projects involving a D2D Network system, BEMS(building energy management system), demand response, design and analysis of energy management systems.

Si-O Seo received the B.S. and M.S. degrees in Computer and Information Science from Korea University, Korea in 2007 and 2009, respectively, and the Ph.D. from Korea University in 2015. In 2015 he joined the BK21 plus team from Department of Computer and Information Science at Korea University, Sejong City, where he is a research professor. His research interests include building energy management systems, demand forecasting, electric vehicle charging system, and design and analysis of energy management systems.

Seung-Yeon Kim received the Ph.D. degree in electronics and information engineering in 2012 from Korea University, Korea. He is currently an assistant professor in the Department of Computer and Information Science. He has participated in various research projects involving an IMT-Advanced system, cognitive radio, and interference management. His research interests include performance evaluation of communication networks.

Choong-Ho Cho received B.S. and M.S. degrees in industrial engineering from Korea University in 1981 and 1983, respectively. He received M.S. and Ph.D. degrees in computer science from the Institute National des Sciences Appliques, Lyon, France, in 1986 and 1989, respectively. He was an assistant professor at Sooncheunhyang University from 1990 to 1994, and is currently a professor at Korea University. He was a visiting professor at university of Washington from 2001 to 2002. His research interests include IT convergence technology, Building Energy Management System, 4G/5G mobile/wireless networks, D2D communication, small cell and SDN. 